\title{
Christine Niederberger Betton
}

Rosa Ma. Reyna Robles

\section{OpenEdition \\ Journals}

Edición electrónica

URL: http://journals.openedition.org/jsa/2219

DOI: 10.4000/jsa.2219

ISSN: 1957-7842

Editor

Société des américanistes

\section{Edición impresa}

Fecha de publicación: 1 enero 2001

Paginación: 401-405

ISSN: 0037-9174

\section{Referencia electrónica}

Rosa Ma. Reyna Robles, « Christine Niederberger Betton », Journal de la Société des américanistes [En línea], 87 | 2001, Publicado el 22 junio 2009, consultado el 15 septiembre 2020. URL : http:// journals.openedition.org/jsa/2219 


\section{Christine NIEDERBERGER BETTON}

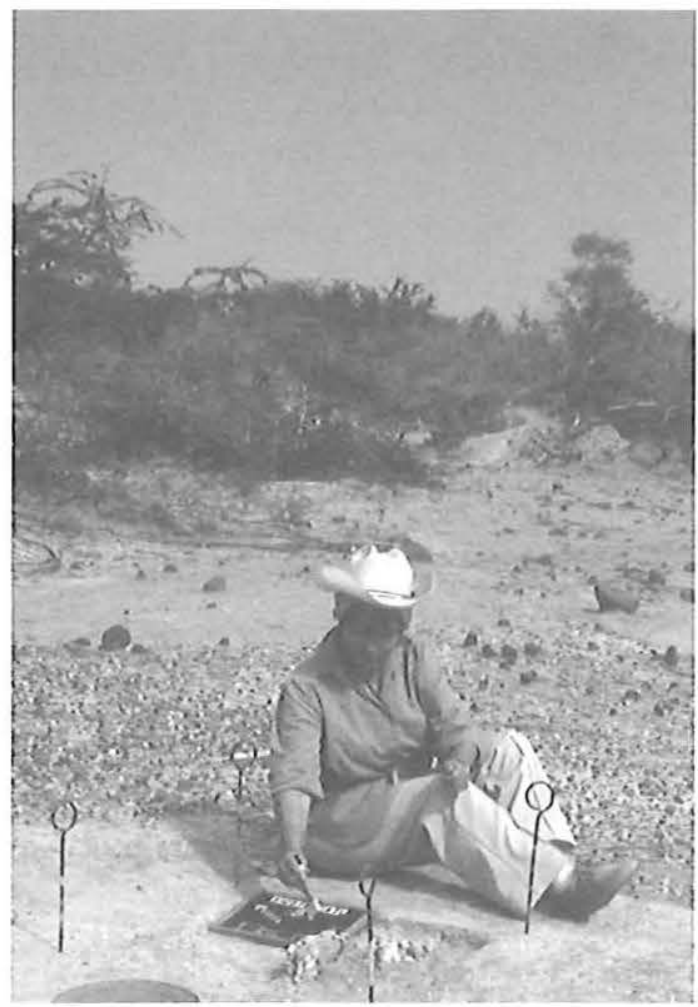

Christine Niederberger sur le site de Xicotencatl dans la " Vallée du Mammouth » (Tamaulipas) en 1984. Photo: Ma. Rosa Avilez

Christine nació en Burdeos, Francia, pero gran parte de su vida adulta la pasó en México, país al que amó profundamente.

Sus padres fueron Roger Betton y Linka Lowczynski, a quienes siempre profirió un gran cariño y respeto. Su educación secundaria la realizó en l'École de la Légion d'honneur. Más tarde, en 1954 y 1955, inició sus estudios superiores en l'École nationale des langues orientales, estudios que interrumpió debido a los numerosos viajes que realizaba al extranjero con su familia.

En México, de 1965 a 1968, cursó la carrera de arqueología en la Escuela Nacional de Antropología e Historia y en 1969 excavó en la ladera del cerro de Tlapacoya, en el predio llamado Zohapilco, bajo la dirección del Mtro. José Luis Lorenzo. 
Con los resultados de dicha excavación, en 1974 presentó su tesis Zohapilco. Cinco milenios de ocupación humana en un sitio lacustre de la cuenca de México. Años después, en 1981, obtuvo el doctorado de Estado en l'École des hautes études en sciences sociales de París con la tesis Paléopaysages et archéologie pré-urbaine du bassin de Mexico, bajo la dirección de Jean Guilaine.

Su investigación como arqueóloga la dedicó a dos temas que la apasionaron : las culturas del Preclásico o Formativo, en especial las de la época olmeca, y la arqueología de Guerrero.

Sus investigaciones siempre fueron de excelencia ; con gran acuciosidad seleccionaba los sitios a excavar, con enorme cuidado recogía y registraba cada una de las evidencias y datos contextuales, con gran paciencia los analizaba y estudiaba y con sobresaliente soltura escribía los resultados en un lenguaje preciso y elegante. Ninguna concha o huesecillo dejó de ser identificado, ninguna roca, ningún tiesto, ninguna semilla o polen, pues siempre se apoyó en los análisis y resultados de diversos especialistas, cuidando fechar los varios estratos y contextos por medio de radiocarbono.

Su tesis de la ENAH fue publicada por el INAH en 1976. Esta importante obra marcó un parte aguas en el conocimiento y secuencia ocupacional de la cuenca de México. En Zohapilco logró establecer claramente que las ocupaciones relacionadas a materiales de estilo olmeca precedían a los niveles con materiales Zacatenco y El Arbolillo, revirtiendo así la secuencia y cronología hasta entonces aceptadas.

Las excavaciones de Christine Niederberger en Zohapilco-Tlapacoya desembocaron también en otra aportación mayor que ella dio a conocer en particular en un artículo famoso publicado por la revista Science. En los primeros niveles de la ocupación humana del sitio (sus fases Playa 1 y Playa 2, entre 6000 y 4500 antes de nuestra era) en efecto, detectó indicios de actividades multiestacionales en un contexto netamente residencial. Esto nos habla de un probable sedentarismo temprano de las poblaciones, anterior al desarrollo de la agricultura. De esta manera quedaba resforzada la hipótesis de un modelo de evolución muy distinto del establecido en el valle de Tehuacan donde los experimentos sobre varios vegetales anteceden mucho al modo de vivir sedentario.

Su segunda obra, verdaderamente monumental, se desprende de su tesis de doctorado. Fue publicada en 1987 por el Centre d'études mésoaméricaines et centraméricaines (CEMCA) en dos volúmenes. En ella dejó plasmado uno de los trabajos más sólidos que se han producido en torno a las culturas preclásicas, no sólo de la cuenca de México sino de toda la naciente Mesoamérica, o América media, como prefería llamarla. Allí retoma el análisis a profundidad del medio ambiente y la geografía de su primer trabajo, añadiendo los resultados de cada excavación arqueológica efectuada hasta entonces en la cuenca, en especial de sitios pre-Cuicuilco ; incluso, con la aprobación de los autores, reprodujo datos inéditos que incluyó en forma de «fichas », además de resumir los datos publicados de todos los sitios preclásicos de las diferentes regiones mesoamericanas.

Una vez establecida la edad de sitios y materiales abordó temas más profundos relacionados con el carácter y naturaleza de las sociedades del Formativo, argumentando consistentemente que no eran igualitarias, como comúnmente se les concep- 
tuaba, sino altamente jerarquizadas, sociedades que llegaron a conformar capitales regionales, o " caput non urbs », como les llamó.

El fenómeno olmeca lo entendía sólo en dos sentidos : como una civilización y como un estilo. De alli se desprenden dos conceptos fundamentales, íntimamente relacionados, que defendió siempre : que los objetos de estilo olmeca presentes en numerosos sitios del Preclásico o Formativo eran creación de una civilización multiétnica y plurilinguística sincrónica, distribuida en un amplio territorio, la naciente Mesoamérica, que se identifica a través de un estilo peculiar panmesoamericano, reflejo de un sistema compartido de creencias, y no producto de la difusión a partir del « Olmec hearthland » de la Costa del Golfo, refutando así esta posición unicentrista y unilateral y otorgando su justo valor a sociedades de otras regiones, poseedoras de estructuras económicas, políticas y administrativas complejas que jugaron un papel activo y creador dentro de la densa trama de intercambios interregionales.

La obra de Christine Niederberger es amplia y siempre de gran calidad. Hasta el último momento escribió con gran conocimiento y profunda sensibilidad; tres de sus trabajos postreros sobre la arqueología de Guerrero pronto serán publicados.

La lamentable pérdida de Christine deja un gran vacío en la investigación arqueológica de excelencia, pero también en las numerosas amistades que supo cultivar en vida. Siempre la recordaremos como una persona culta, inteligente, bondadosa, generosa y honesta. Le sobrevive su esposo, Jean-Marie Niederberger, al que amó entrañablemente.

Querida amiga, descansa en paz.

\section{Rosa Ma. Reyna Robles}

Instituto nacional de antropología e historia, México

\section{PUBLICACIONES}

1969. — « Paleoecología humana y playas lacustres postpleistocénicas en Tlapacoya », Boletín del INAH, n ${ }^{\circ} 37$, pp. 19-24, INAH, México.

1970. - « Excavations at Tlapacoya, Mexico. Cultural Remains II », XXV Annual Meeting, Society for American Archaeology, Departamento de Prehistoria, INAH, México.

1974. — « Inicios de la vida aldeana en América Media », Historia de México, vol. I, pp. 93-130, Salvat, Barcelona-México.

1975. — « Excavaciones en Tlapacoya-Zohapilco », XLI Congreso Internacional de Americanistas, Actas, vol. I, pp. 403-411, INAH, México.

1976. - Zohapilco. Cinco milenios de ocupación humana en un sitio lacustre de la Cuenca de México, Colección Científica, $\mathrm{n}^{\circ}$ 30, INAH, México.

s.f. - « Modalités d'instauration de la vie sédentaire dans le Sud du Bassin de Mexico », Communication (4 mars 1976), Société des Américanistes, Musée de l'Homme, Paris.

1979. - «Early Sedentary Economy in the Basin of Mexico », Science, vol. 203, pp. 132-142, American Association for the Advancement of Science, Washington.

1980. — « Dos casos de desarrollo agrícola y utilización del medio ambiente : Tlapacoya, Edo. de México y Coxcatlán, Puebla », Seminario de Ecología Agrícola (19-24 de Octubre), Universidad Autónoma Metropolitana-Xochimilco, México. 
1981a. - «Paleopaisajes de la Cuenca de México », Atlas de la Ciudad de México, pp. 28-29, Secretaría de Recursos Hidraúlicos, México.

1981b. - « De la prehistoria a las primeras capitales en la Cuenca de México », Atlas de la Ciudad de México, pp. 30-31, Secretaría de Recursos Hydraúlicos, México.

1981c. - «Les premières capitales (Caput non Urbs) du Bassin de México », Xe Congrès de l'Union Internationale des Sciences Préhistoriques et Protohistoriques, vol. 16, pp. 151-170, México.

1985a. - "Sédentarisation et paléoenvironnements en Amérique Moyenne », Grand atlas de l'archéologie, pp. 342-343, Encyclopaedia Universalis, Paris.

1985b. - " La civilisation olmèque ou la naissance de la Mésoamérique », Grand atlas de l'archéologie, pp. 344-345, Encyclopaedia Universalis, Paris.

1985c. — « Les communautés pré-urbaines d'Amérique Moyenne », Grand atlas de l'archéologie, pp. 346-347, Encyclopaedia Universalis, Paris.

1986. — « Excavación de un área de habitación doméstica en la capital "olmeca ” de Tlacozotitlán, Guerrero », Arqueología y Etnohistoria del Estado de Guerrero, pp. 81-103, Primer Coloquio de Arqueología y Etnohistoria del Estado de Guerrero, (Noviembre 1984), INAH - Gobierno del Edo. de Guerrero, México.

1987a. - Paléopaysages et archéologie pré-urbaine du Bassin de Mexico, Collection Études Mésoaméricaines, I-11, 2 vol., 855 p., Centre d'études mexicaines et centraméricaines, México.

1987b. - " Middle America : from the Beginning of a Sedentary Life to the Rise of the First Regional Centers ", XIe Congrès de l'Union Internationale des Sciences Préhistoriques et Protohistoriques, Römisch-Germanisches Zentralmuseum, Mainz.

1988. - « La arqueología sobre el período formativo y la época pre-Cuicuilco en la Cuenca de México », La Antropología en México, vol. 14, pp. 59-80, C. García Mora (coord.), Colección Biblioteca del INAH, INAH, México.

1990. - «L'imaginaire collectif et l'art sacré de la Mésoamérique ancienne », table ronde présidée par J. Soustelle, Exposition Art précolombien du Mexique, Grand Palais, Paris.

1994. - Introducción y presentación de la obra « Entre lagos y volcanes : Chalco-Amecameca pasado y presente », UAM-Ixtapalapa, Colegio Mexiquense (Febrero 1994), Toluca.

1995. - «Early Mesoamerica : a non-diffusionist perspective from Central Highlands and Western Mexico ", Princeton Symposium on the Olmec, (December 16, 1995), International Symposium organized by The Art Museum, Princeton University in conjunction with the exhibition The Olmec World. Ritual and Rulership, Princeton.

1996a. - "Mesoamerica : Genesis and First Developments ", History of Humanity. Scientific and Cultural Development, vol. II : From the Third Millenium to the Seventh Century BC, pp. 462-475, A. H. Dani \& J.-P. Mohen (ed.), UNESCO, Paris/Routledge, Londres.

1996b. - «The Basin of Mexico : a Multimillenial Development toward Cultural Complexity ", Olmec Art of Ancient Mexico, pp. 83-93, E. P. Benson \& B. de la Fuente (ed.), National Gallery of Art, Washington, \& H. N. Abrams, Inc., New York.

1996c. - «Olmec Horizon Guerrero », Olmec Art of Ancient Mexico, pp. 95-103, E. P. Benson \& B. de la Fuente (ed.), National Gallery of Art, Washington, \& H. N. Abrams, Inc., New York.

1996d. - «Paisajes, economía de subsistencia y agrosistemas en Mesomérica a principios del siglo XVI ", Temas mesoamericanos, pp. 11-50, S. Lombardo \& E. Nalda (ed.), INAH- 
Dirección General de Publicaciones del Consejo Nacional para la Cultura y las Artes, México.

1997. - " An approach to Paleolithic Technology and Art in Middle America », The Dictionary of Art, MacMillan Publishers, Londres.

1998a. - "Las sociedades mesoamericanas antiguas », Historia de América Latina, vol. I, capítulo 6, UNESCO, Paris.

1998b. — « Presentación » de la publicación de R. Ma. Reyna Robles y L. González Quintero, titulada Rescate arqueológico de un espacio funerario de época olmeca en Chilpancingo, Guerrero, Colección Científica n 382 , INAH, México.

2000. - « Tres años antes de que se apague para siempre el sonido del tambor de Mato-Topé o El viaje del príncipe de Wied en el valle del Misuri : 1833-1834 », Nómadas y sedentarios en el Norte de México, Coloquio en Homenaje a la Dra Beatriz Braniff (2-6 de Octubre de 1995), Universidad Juárez del Estado de Durango, Durango.

2000. - « Ranked Societies, Iconographic Complexity and Economic Wealth in the Basin of Mexico towards 1200 BC ", Symposium on Olmec Art and Archaeology in Mesoamerica : Social Complexity in the Formative Period (20-21 September 1996), Center for Advanced Study in the Visual Arts, National Gallery of Art, Washington.

En prensa

s.f.1. - "Antiguos paisajes de Guerrero y el papel de su fauna en las creencias míticas », Investigaciones arqueológicas en el estado de Guerrero : Balance y perspectiva a principios del siglo XXI.

s.f.2. - "Nacar, " jade " y cinabrio : Guerrero y las redes de intercambio en la Mesoamérica ", Investigaciones arqueológicas en el estado de Guerrero : Balance y perspectiva a principios del sigl XXI.

s.f.3. - « Formative figurines from Central Mexico : Sensitive markers of the post-Covarrubias new chronology and salient testimonies of ancient behavior and beliefs », Early Figurines in Mesoamerica, John E. Clark (ed.), Ancient Mesoamerica, (William Fowler).

s.f.4. - con Louis Bazin : «Les Amériques », Introduction au Vol. III, History of Humanity, UNESCO, Paris.

s.f.5. - con Rosa Ma. Reyna : «Alto al saqueo en la cuenca del Balsas : una llamada de auxilio ", Investigaciones arqueológicas en el estado de Guerrero : Balance y perspectiva a principios del siglo XXI. 